This item was submitted to Loughborough's Research Repository by the author.

Items in Figshare are protected by copyright, with all rights reserved, unless otherwise indicated.

\title{
Use of real time creep force estimation data for assessment of low adhesion in the wheel/rail contact
}

PLEASE CITE THE PUBLISHED VERSION

PUBLISHER

The Institution of Engineering and Technology (IET) @ The authors

VERSION

AM (Accepted Manuscript)

LICENCE

CC BY-NC-ND 4.0

\section{REPOSITORY RECORD}

Ward, Christopher P., Roger M. Goodall, Roger Dixon, and Guy Charles. 2019. "Use of Real Time Creep Force Estimation Data for Assessment of Low Adhesion in the Wheel/rail Contact". figshare. https://hdl.handle.net/2134/9211. 
This item was submitted to Loughborough's Institutional Repository (https://dspace.lboro.ac.uk/) by the author and is made available under the following Creative Commons Licence conditions.

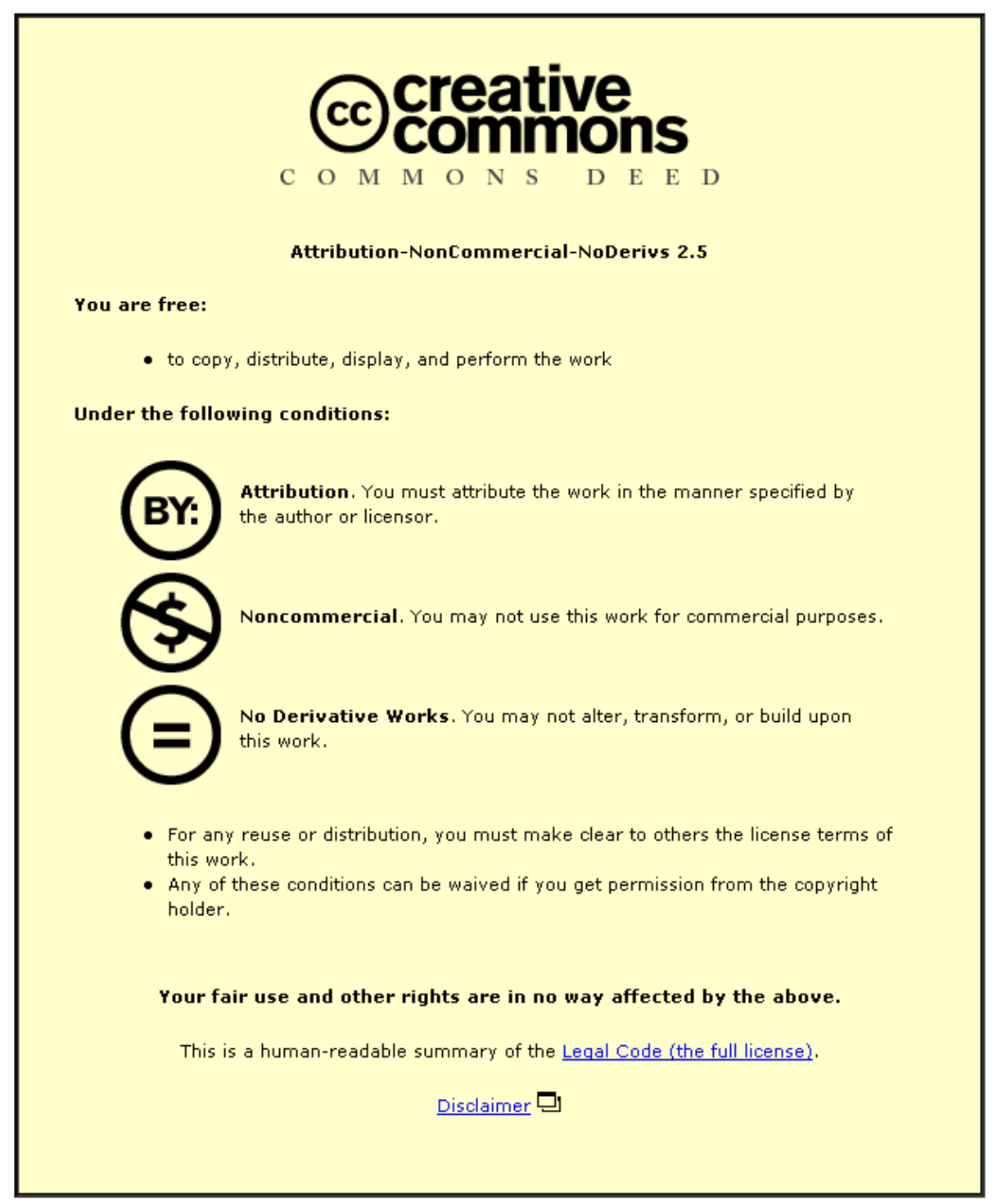

For the full text of this licence, please go to: http://creativecommons.org/licenses/by-nc-nd/2.5/ 


\title{
USE OF REAL TIME CREEP FORCE ESTIMATION DATA FOR ASSESSMENT OF LOW ADHESION IN THE WHEEL/RAIL CONTACT
}

\author{
C.P. Ward*, R.M. Goodall*, R. Dixon* and G. Charles ${ }^{\dagger}$ \\ *Control Systems Group, School of Electronic, Electrical and Systems Engineering \\ Loughborough University, UK \\ c.p.ward@lboro.ac.uk, r.m.goodall@lboro.ac.uk, r.dixon@lboro.ac.uk \\ ${ }^{\dagger}$ Department of Mechanical, Materials and Manufacturing Engineering, \\ University of Nottingham, UK \\ guy.charles@nottingham.ac.uk
}

Keywords: Condition monitoring, Kalman-Bucy filtering, real-time estimation, creep forces, system identification

\begin{abstract}
Low adhesion in railway vehicle wheel/rail contact can create large cost and punctuality issues for train operators and users. There is currently a lack of real time knowledge of the running conditions on networks, therefore the work reported here proposes a method of detecting dynamically the adhesion conditions underneath a rail vehicle using modest cost sensors and advanced model based monitoring of the creep forces. The estimated creep forces are then post processed to give an estimation of the current adhesion condition.
\end{abstract}

\section{Introduction}

Areas of low adhesion in the wheel/rail interface can create large cost and punctuality issues for train operators and users. These often ephemeral areas manifest in timetables needing to be rescheduled for reduced station stop and signal approach speeds, especially in autumn when the problem is at its height. This mitigation can have the effect of reducing capacity on already crowded networks. At present there is a lack of real time knowledge of the current adhesion condition across the network, and this is being addressed via the RSSBfunded research project T959 "On Board Detection of Low Adhesion", sponsored by the UK's Technical Strategy Leadership Group (TSLG) and the Vehicle-Track Systems Interface Committee (VTSIC).

The concept of the project is to determine the current condition of adhesion below a rail vehicle in real time under normal running conditions using modest cost sensors and advanced processing methods. This is conceptually different from WSP (wheel slide protection) and TC (traction control) systems as it does not require a high slip condition to be triggered in order to identify an area of low adhesion. Previous studies [3,15] established firstly that when the adhesion drops to a level sufficient to cause operational problems the effect is seen in the dynamic response of the bogie to track alignment irregularities, and secondly that in concept generalised creep forces can be extracted from inertial sensors distributed around a rail vehicle with this data processed by advanced model based filters such as the well known Kalman-Bucy method [8]. It was shown in [10] that, as the wheel/rail interface adhesion level drops, the initial slope of a creep curve reduces, which manifests itself as a reduction in the creep forces produced even before the forcecreep characteristic saturates. Consequently, if creep forces can be estimated the adhesion condition can be inferred.

However creep forces in their basic form cannot be used to determine the adhesion conditions and these signals require post processing due to their dependance upon lateral track irregularity size and vehicle forward velocity. This paper therefore firstly recaps the plan view simulation modelling and the creep force estimation method via Kalman-Bucy filtering used in this initial phase of the testing. It then explores a number of post processing techniques applied to the estimated creep force signals; these are: wavelet analysis; output regressor identification; and how these might be combined into a global adhesion scheme. Use of stored track data to improve the determination of adhesion conditions is also explored. Finally details are given on how these concepts will be tested on a full scale vehicle.

\section{System modelling}

The aim of the analysis is to initially determine the creep forces present in the wheel-rail contact during normal running. Therefore before experimentation is undertaken a sufficiently detailed simulation model is required. The details of the modelling of the simulation model have been covered in detail before in $[15,16]$ and are briefly recapped here.

The model is considered only in plan-view, i.e. lateral and yaw dynamics, as the coupling with longitudinal and vertical dynamics can be neglected as they are not important for the creep force estimation [17]. The model is thought of in two sections, the nonlinear wheel/rail contact interaction and the 
Newtonian dynamics of the vehicle's primary and secondary suspension systems.

\subsection{Contact modelling}

Use is made of the Polach [11] method of creep force modelling due to its ability to model variations in the initial creep slope that can't be accommodated with the linear Kalker theory [7]. The combined lateral and longitudinal creep force (excluding spin effects) are calculated as

$$
F=\frac{2 Q \mu}{\pi}\left(\frac{k_{A} \varepsilon}{1+\left(k_{A} \varepsilon\right)^{2}}+\arctan \left(k_{s} \varepsilon\right)\right)
$$

where $k_{A}$ is the area of adhesion and $k_{s}$ is the area of slip, $Q$ is the wheel load, and $\varepsilon$ is the gradient of the tangential stress in the area of adhesion. The friction coefficients rely upon the slip velocity as

$$
\mu=\mu_{0}\left[(1-A) e^{-B w}+A\right]
$$

where $w$ is the total slip velocity, $A$ is the ratio of the limit friction coefficient at the infinite slip velocity $\mu_{\infty}$ to the maximum friction coefficient $\mu_{0}$, and $B$ is the coefficient of exponential friction decrease.

Using this method specific adhesion conditions can be set using five parameters $\left(k_{A}, k_{S}, A, B, \mu_{0}\right)$ mentioned above to create a model of varying initial creep slopes. Values for these parameters can be found in [15] for the four test conditions used: dry; wet; low adhesion; and very low adhesion. Figure 1 shows the effect of these variations in adhesion modelling upon the dynamic performance of the modelled vehicle. It shows that as the adhesion condition reduced the creep forces produced for the same track irregularity also reduce, meaning that a drop in adhesion should be detectable.

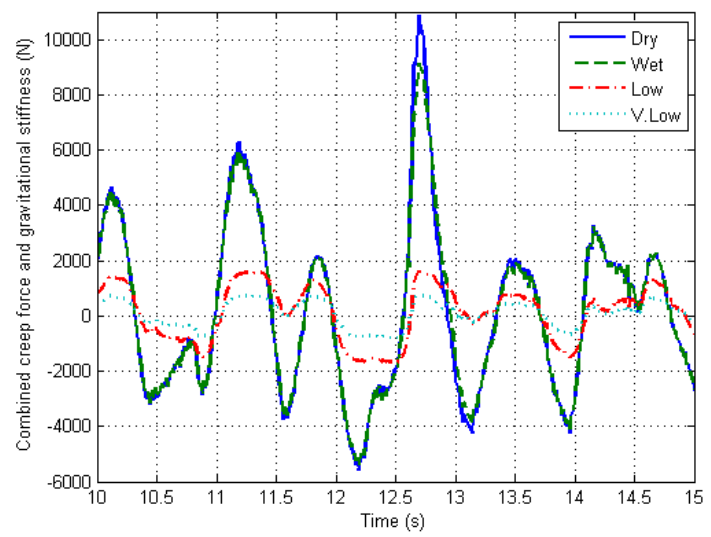

Figure 1: Simulation of the creep force variation

\subsection{Vehicle component modelling}

This study uses a full vehicle plan-view model of the British Mk.3 coaching stock. The model therefore consists of one leading bogie with two wheelsets, one trailing bogie with two wheelsets and the vehicle body. The full details of the vehicle and suspension modelling can be found in [15].

\section{Creep force estimation}

The well-known continuous time Kalman-Bucy filter [8] is used to estimate the creep forces present in the wheel-rail contact. Initial studies [2] attempted to estimate the separate contact forces individually. However it was shown that the filter as designed was not able to distinguish between longitudinal, lateral and left and right contact patch forces. Therefore the creep forces and gravitational stiffness forces are estimated as combined states. Again, details of the filter formation can be found in [15].

In application the Kalman-Bucy filter gains will be statically calculated before application but the filter will be required to work with different adhesion characteristics present on the railhead, therefore a series of tests were undertaken for various adhesion conditions to test the efficacy of the estimator. Initially these were at static levels of adhesion at the four levels set for the study: dry; wet; low; and very low. Figure 2 shows a section of estimated creep torque data for the same track irregularity for all four of the adhesion conditions.

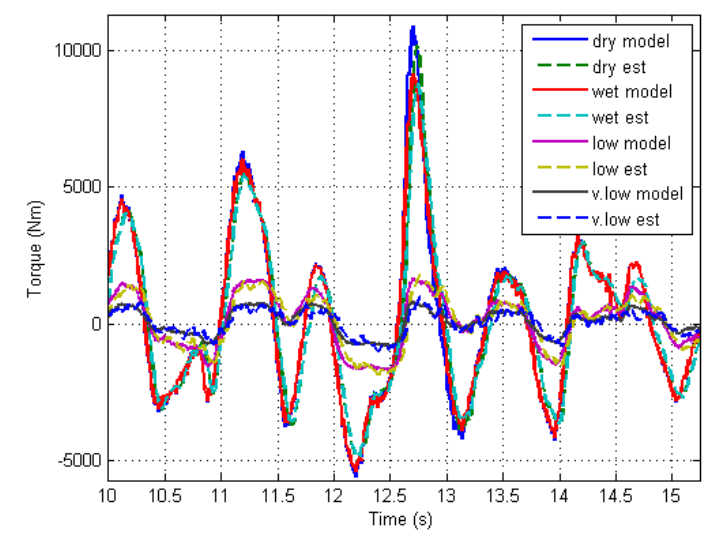

Figure 2: Creep estimation at constant adhesion levels

This shows that the filter can adapt well to different adhesion conditions. It estimates the combined creep and gravitational stiffness forces with coefficient of determination $\left(\mathrm{R}^{2}\right.$ values as described in [9]) of approximately $70 \%$ to $90 \%$ [16]. However these creep forces vary with vehicle speed and the size of the lateral track irregularity. A method of interpreting the time-based creep forces into usable interpretations of the adhesion level is required; several suggested methods are covered in the following section.

\section{Creep force post processing}

Creep forces alone cannot be used to assess the level of adhesion due to their dependence upon the lateral track irregularity size and the vehicle speed. The basic concept of analysing the creep force estimates to produce a meaningful understanding of adhesion level is shown in the schematic in Figure 3. The basic algorithm flow is: vehicle based measurements are used in the estimation of the creep forces; 
the estimates are then 'post-processed' by some other mathematical means; the output is interpreted as a level of adhesion. There is the potential that this post processing algorithm may utilise information about: the vehicle speed; the track irregularity; or data from previous traversals of the same section of track. However it would be more acceptable if these additional sources of information were not utilised as this would enable the method to be completely 'standalone' and therefore independent of any end user maintenance.

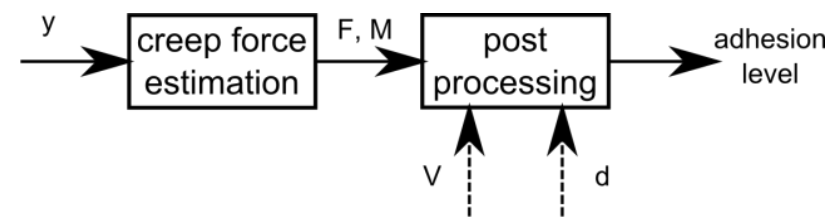

Figure 3: Post processing concept flow

A number of analysis techniques were assessed, these included: PSD analysis [2]; PCA (principal component analysis) [6]; wavelet analysis [13]; and use of system identification techniques [9]. Each of these offered varying degrees of resolution and sensitivity to track irregularity size. The most successful outcomes are shown below.

\subsection{Wavelet transform analysis}

Wavelet transforms (WT) are used in a number of applications from data compression to interrogating the frequency components of signals. More commonly a signal's frequency components are analysed by a Fourier transform (FT). FT however loses time specific information as its assumptions are based upon stationary signals in which the frequency components are constant.

FT can be applied to moving 'windowed' data to track frequency and amplitude changes in time with signals such as those produced by the creep force estimation method. The window chosen must be long enough to allow the important frequencies to be analysed, though this can add time delay into the analysis such as on the creep force data application, meaning the adhesion level could be detected several seconds late. It was first suggested in [2] to look at the peak of a PSD signal which should be around the $1 \mathrm{~Hz}$ area, coincident with the vehicle modes. This however proved unsatisfactory for a range of track irregularities and with parameters for the Mk.3 coach.

The WT offers to do better than this. It provides the same basic information (changing frequencies with time) but the resolution in the time axis increases with frequency (that is analogous to using a smaller window of data for the high frequencies and a larger one for the low frequencies). Hence with a WT more useful information is kept compared to the FT and the potential benefit here is that the frequency components of the creep force signal can be tracked as they change and these changes will be seen at the highest possible resolution. Full details of the analytical method of wavelet analysis can be found in [13].
The WT technique was applied here to moving window data of the creep force and moment estimations. The analysis uses 20 different wavelet 'frequencies', most of which do not demonstrate any significant change. However the $16^{\text {th }}$ wavelet shown in Figure 4 (b) demonstrates that, as the adhesion experiences a step reduction at 15 seconds, so does the amplitude of this particular signal. To make the signal more visible the variance of the signal is extracted and is seen in Figure 4 (c), however this can be seen to add a time delay in the signal equivalent to the 3 second size of the sample window. This clearly demonstrates the drop in the signal level, threshold levels for which can set to determine the adhesion level but the variance window needs careful selection: too small and the variance reacts to all of the small changes; too long and changes take a long time to register.
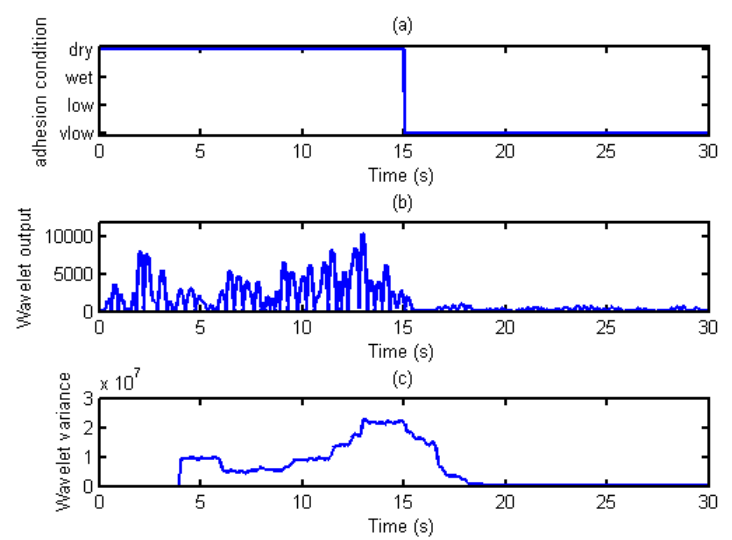

Figure 4: WT adhesion step test: (a) adhesion change; (b) $16^{\text {th }}$ WT output; (c) WT 3 second time window variance

However Figure 5 shows that the wavelet analysis overlap for different levels of track irregularity at constant adhesion conditions, therefore if threshold level are set to differentiate between adhesion levels 'false triggers' may occur for different levels of track irregularity.

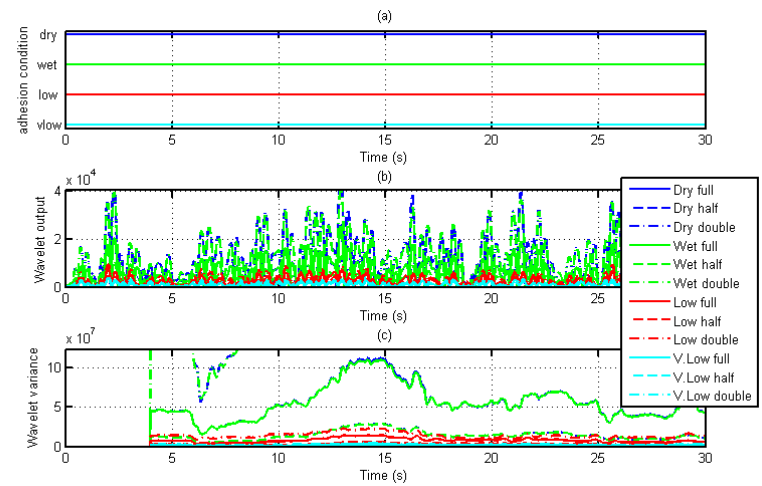

Figure 5: WT constant adhesion levels: (a) adhesion levels; (b) $16^{\text {th }}$ WT output; (c) WT 3 second time window variance

However if there is some knowledge of the track irregularity size this can be used to scale the wavelet output, and an example of this is shown in Figure 6 where the wavelets are now separated for the low and very low conditions for various 
track irregularity sizes. This refinement however has the detriment of line knowledge being required so the system would now require expert user input and upkeep and precise synchronisation with the vehicle location.

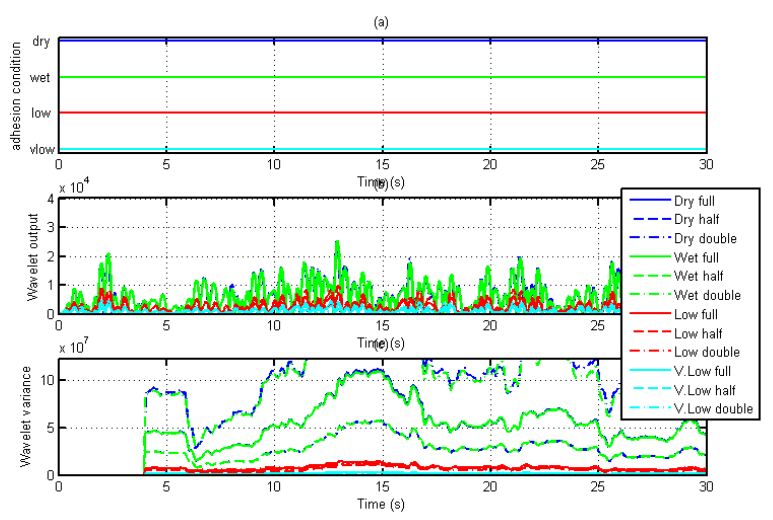

Figure 6: WT constant adhesion levels with scaling: (a) adhesion level; (b) $16^{\text {th }}$ WT output; (c) WT 3 second time window variance

\subsection{Output identification regressor analysis}

System identification is a concept that was first used to create dynamic models of system from collected data instead of first principles analysis [9]. The essential principle is to minimise the sum squared error of the residual vector

$$
r_{t}=y_{t}-\hat{y}_{t}
$$

where $r_{t}$ are the residuals, $y_{t}$ is the observed data and $\hat{y}_{t}$ is the predicted data. The standard system equation in matrix form is

$$
y=X \theta+\varepsilon
$$

where $\mathrm{y}$ is the measure output, $\mathrm{X}$ is the user selected matrix of parameter regressors, $\theta$ the selected parameter values and $\varepsilon$ is the equation error. The least squares solution is of the form

$$
\left(X^{T} X\right) \theta=X^{T} y
$$

This can be rearranged to the easily handled form

$$
\theta=\left(X^{T} X\right)^{-1} X^{T} y
$$

The method is adapted here to identify frequency based changes in the data from the creep force estimation algorithms, the concept being to identify the creep force signal from regressor data of the creep forces. The eigenvalues from this model can then be assessed to determine if any significant changes in the adhesion level have occurred.

The model formation for the identification is shown as

$$
\hat{F}_{t}=\theta_{1} F_{t-1}+\theta_{2} F_{t-2}+\cdots+\theta_{n} F_{t-n}
$$

where $\hat{F}_{t}$ is the creep force estimate through the identification process, $F_{t-n}$ is the time regressor at sample $n$ of the estimated creep force from the Kalman filter algorithm, and $\theta_{n}$ is the estimated parameter for the corresponding regressor signal.
In this example a first order model is used with a corresponding single eigenvalue. Figure 7 shows the output for the step test change in adhesion level as used in the previous example for three sizes of track irregularity: full scale; double scale; and half scale.

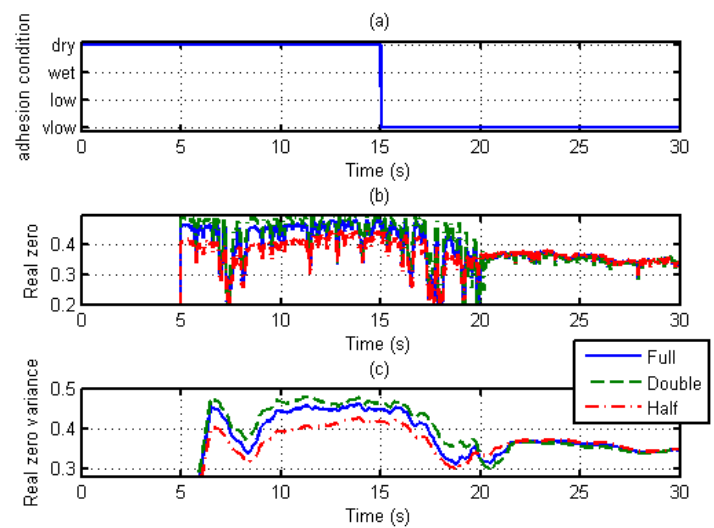

Figure 7: Regressor identification analysis: (a) adhesion change; (b) raw eigenvalues; (c) eigenvalue variances

Subplot (b) shows the real part of the eigenvalues of the first order polynomial identification for a five second window of data. The graphs are quite noisy and difficult to interpret, but show a definite change as the adhesion condition reduces. This signal is subsequently time averaged over a two second window and is shown in subplot (c). This more clearly shows the change in the frequency content of the signal, therefore as with the other techniques thresholds for this change can be set to determine the adhesion condition. As with the wavelet technique careful selection is required for the size of the sample windows. The figure also shows that the above trends are repeated for a range of track irregularity levels.

\subsection{Estimation of traction coefficients}

The previous techniques have all relied in essence upon user based knowledge of the system to impose thresholds on processed data to determine changes in the adhesion conditions that may be dependent upon a particular section of track. The concept behind this post-processing technique is to use the estimated creep forces and some knowledge of the track (this may be as time data, frequency data, or some frequency based density assumptions) to identify linearised traction coefficients.

This technique again uses the system identification ideas of the previous section, with linearised models of the creep forces. Using a simplified linear model from [4] the lateral and yaw creep forces for the front wheelset can be represented as

$$
F_{F F}=2 f_{22} \psi_{F F}-\frac{2 f_{22}}{V} \dot{y}_{F F}
$$




$$
\begin{aligned}
M_{F F}= & -\frac{2 l \lambda f_{11}}{r_{0}}\left(y_{F F}-d_{F F}\right) \ldots \\
& -\frac{2 l^{2} f_{11}}{V} \dot{\psi}_{F F}
\end{aligned}
$$

$f_{11}$ is the longitudinal creep coefficient, $f_{22}$ is the lateral creep coefficient, $\lambda$ is the linearised conicity of the wheelset, $l$ is the half width of the bogie, $F_{F F}$ and $M_{F F}$ are the lateral creep force and yaw creep torque respectively, $y_{F F}$ is the lateral position of the wheelset, $d_{F F}$ is the lateral track irregularity, $\psi_{F F}$ is yaw position of the wheelset, $V$ is the forward vehicle speed and $r_{0}$ is the rolling radius of the wheels. The assumption for the parameterisation model is that

$$
\begin{aligned}
& F_{F F}=\theta_{1} \psi_{F F}-\theta_{2} \dot{y}_{F F} \\
& M_{F F}=\theta_{3}\left(y_{F F}-d_{F F}\right)+\theta_{4} \dot{\psi}_{F F}
\end{aligned}
$$

Equation 11 shows that some information of the track irregularity is required. At present it is assumed that this is available as a time signal of the lateral irregularity, but the technique is being developed with a spectral density assumption of the signal. Other geometric knowledge is required of the assumed linearised conicity (which changes with time), and the speed of the vehicle. The creep coefficients can then be found working backwards from the identified parameter values from

$$
\begin{aligned}
& f_{11_{a}}=-\frac{\theta_{3} r_{0}}{2 l \lambda} ; f_{11_{b}}=-\frac{\theta_{4} V}{2 l^{2}} \\
& \hat{f}_{11}=\frac{f_{11_{a}}+f_{11_{b}}}{2} \\
& f_{22_{a}}=\frac{\theta_{1}}{2} ; f_{22_{b}}=-\frac{\theta_{2} V}{2} \\
& \hat{f}_{22}=\frac{f_{22_{a}}+f_{22_{b}}}{2}
\end{aligned}
$$

An example of the analysis for the lateral creep coefficient is shown in Figure 8 for a step reduction in the adhesion level at 15 seconds. The analysis is performed for a 5 second time window for three different levels of track irregularity. It can be seen that a definite reduction in the estimated creep coefficient is seen and that the levels are consistent for all of the track irregularity levels.

\section{Further developments}

The previous section highlighted a number of techniques for the assessment of the estimated creep forces. However a number of other methods are being investigated to provide additional understanding of the adhesion conditions, including the use of multiple model techniques and black box approaches that don't require system models.

\subsection{Multiple model approaches}

Multiple model approaches have been used in many applications for numerous years, such as missile guidance where the dynamics of the system change demonstrably through extreme manoeuvres. The solution is to run multiple filters concurrently with different dynamic conditions. This same concept can be adapted for adhesion estimation by running multiple models with known adhesion conditions, the model which has the lowest residuals is closest to the current adhesion level, [5].

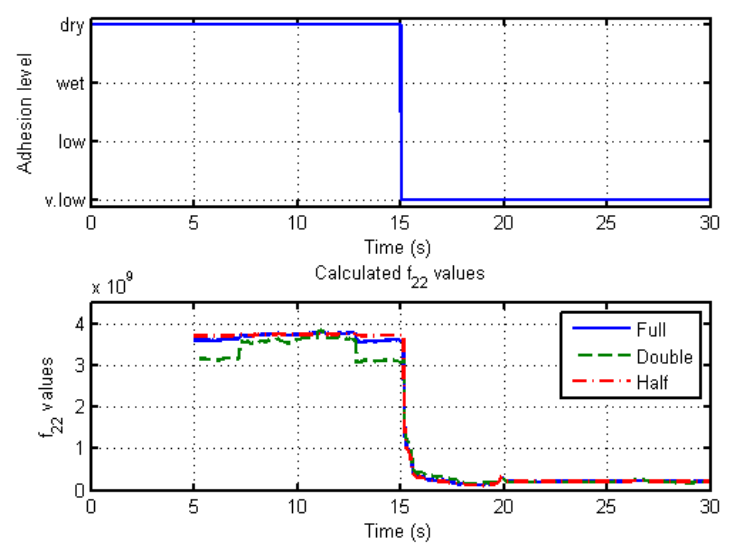

Figure 8: Identification of traction coefficients; (a) adhesion change; (b) estimated lateral creep coefficient

However this is still a rather crude estimation method and the interacting multiple model approach of [1] is an improvement. This gives a statistical understanding of the relevance of each model to the current adhesion conditions through multiple data fusions. The technique has already been applied to adhesion estimation in the automotive field, [14] and is currently being investigated.

\subsection{Black box data analysis}

All of the techniques proposed so far have been model-based and as such rely upon the physics of the system being well understood and intrinsically static. However short and long term changes in adhesion can be detected using many signal processing concepts such as the comparison of data from different traversals of the same section of track using the coefficient of determination [9] or the Sprague and Geers tests [12]. These metrics do not give numerical information about the size of the change in adhesion, but can highlight where a change has occurred in order to validate the outputs of the model based estimators.

\subsection{Technique combinations}

The techniques highlighted so far and those currently being developed all have certain beneficial qualities and also disadvantages. The current thinking is to run a number of the techniques in parallel in order to gain a more robust understanding of the adhesion condition. This concept is shown in Figure 9, with the creep force estimation and post processing shown in combination with the IMM and black box methods. 
The outputs from the individual estimations would then be passed through a fuzzy logic to determine the true adhesion condition. Similar architecture has been used in the estimation of adhesion condition for road vehicles [3].

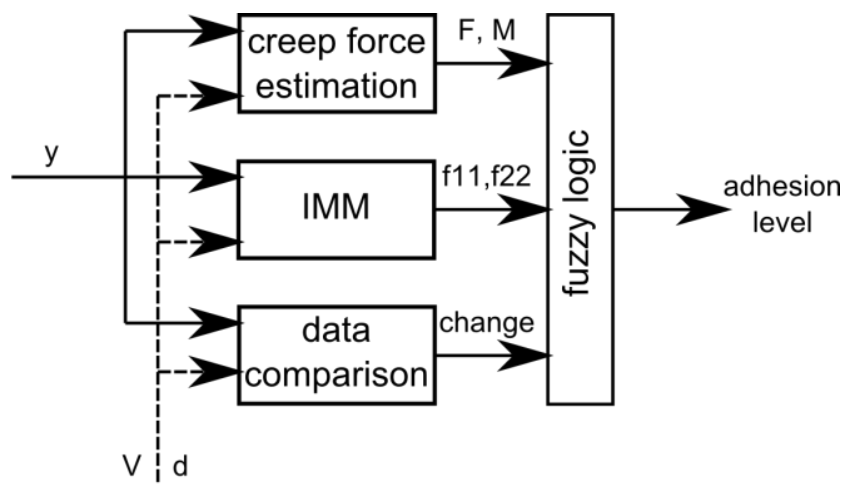

Figure 9: Processing data fusion

\subsection{Further testing}

The techniques demonstrated so far have been applied to simplified system models. The next two stages of project T959 is to apply the techniques firstly to more representative data produced by a multi-bodied dynamics simulation program. This will be provided by a third party supplier so that unknown adhesion conditions will be applied to test the efficacy of the techniques.

If this is successful full scale testing will commence where artificial adhesion conditions are created on a test track and the collected data analysed using a variety of the processing techniques that have been described.

\section{Conclusions}

Low adhesion in the wheel-rail contact of rail vehicles is a large problem. Creep force estimation using low cost inertial sensors is a proposed method for the detection of these events. However these signals cannot be used alone and a number of post-processing methods have been explored (use of wavelets and system identification), some of which require additional expert user inputs such as lateral track irregularities. A number of other methods for the detection of low adhesion were proposed, such as interacting multiple models and data comparison, that could be included in a global estimation scheme to provide a robust solution to the problem. The techniques will be further validated through MBS data and full scale testing.

\section{Acknowledgements}

The authors would like to thank RSSB who manage project T959 with funding provided from the Rail Industry Strategic Research Programme.

\section{References}

[1] H.A.P. Blom and Y. Bar-Shalom. "The interacting multiple model algorithm for systems with Markovian switching coefficients", IEEE Transactions of Automatic Control, volume 33, pp.780-783, (1998)

[2] G. Charles, R.M. Goodall and R. Dixon. "Model based condition monitoring at the wheel-rail interface", Vehicle System Dynamics, volume 46, supplement, pp. 415-430, (2008).

[3] W. Cho, T. Chung and K.Yi. "Estimation of individual tire-road friction coefficients based on tire force information", In proceedings of the $22^{\text {nd }}$ IAVSD symposium, Manchester, paper 44.8, (2011)

[4] V. Garg and R.Dukkipati. "Dynamics of railway vehicle systems", Academic press, Canada, (1984)

[5] I. Hussain and T.X. Mei. "Multi Kalman filtering approach for estimation of wheel-rail contact conditions", In proceedings of the UKACC International Conference on Control, Coventry, UK, (2010)

[6] I. Jolliffe, "Principal component analysis", Springer, Second Edition, (2002).

[7] J. Kalker, "On the rolling contact of two elastic bodies in the presence of dry friction", PhD Thesis, Delft University of Technology, Delft, Netherlands, (1967).

[8] R. Kalman. "A new approach to linear filtering and prediction", Transactions of the ASME - Journal of Basic Engineering, volume 82 (series D), pp. 35-45, (1960).

[9] L. Ljung, "System identification theory for the user", Prentice-Hall, Upper Saddle River, New Jersey, USA, (1999).

[10] T. Pearce and K. Rose, "Measured force-creep relationships and their use in the vehicle response calculation", In proceedings of the $9^{\text {th }}$ IAVSD symposium, Linkoping, (1985).

[11] O. Polach, "Creep forces in simulation of traction vehicles running on adhesion limit", Wear, volume 258(1), pp 992-1000, 2005.

[12] M.A. Sprague and T.L. Geers, "Spectral elements and field separation for an acoustic field subject to cavitation", Journal of Computational Physics, volume 184, pp.149-162, (2003)

[13] G. Strang and T. Nguyen, "Wavelets and filter banks", Wellesley Cambridge Press, Boston, (1997).

[14] H. Tsunashima, "Vehicle and road state estimation using interacting multiple model approach", In proceedings of the $19^{\text {th }}$ IAVSD symposium, Milano, (2005)

[15] C.P. Ward, R.M. Goodall and R. Dixon. "Creep force estimation at the wheel-rail interface", In proceedings of the $22^{\text {nd }}$ IAVSD symposium, Manchester, paper 13.1, (2011)

[16] C.P. Ward, R.M. Goodall and R.Dixon. "Contact force estimation in the railway vehicle wheel-rail interface", In proceedings of the IFAC $18^{\text {th }}$ World Congress, Milano, (2011).

[17] A.H. Wickens. "Fundamentals of rail vehicle dynamics, Guidance and Stability", Swets \& Zeitlinger, Lisse, Netherlands, 2003 